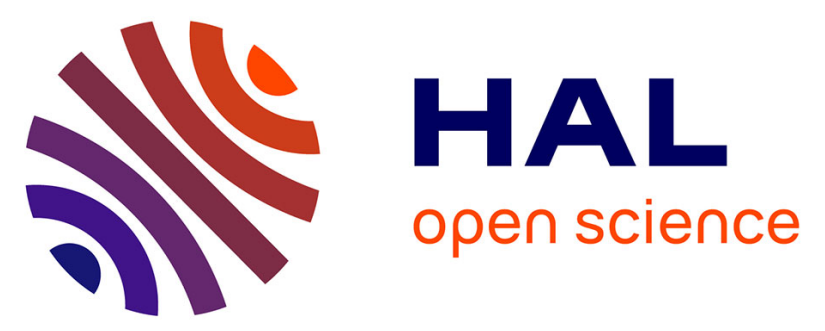

\title{
Design optimisation of a nonlinear energy Sink embedded on a harmonically forced linear oscillator: theoretical and experimental developments
}

Etienne Gourc, Guilhem Michon, Sébastien Seguy, Alain Berlioz

\section{- To cite this version:}

Etienne Gourc, Guilhem Michon, Sébastien Seguy, Alain Berlioz. Design optimisation of a nonlinear energy Sink embedded on a harmonically forced linear oscillator: theoretical and experimental developments. ASME International Design Engineering Technical Conferences \& Computers and Information in Engineering Conference, DETC2012-71014, 12-15 août 2012, Chicago, IL, États-Unis, Aug 2012, Chicago, United States. pp.589-598, 10.1115/DETC2012-71014 hal-02186317

\section{HAL Id: hal-02186317 \\ https://hal.science/hal-02186317}

Submitted on 5 Jul 2021

HAL is a multi-disciplinary open access archive for the deposit and dissemination of scientific research documents, whether they are published or not. The documents may come from teaching and research institutions in France or abroad, or from public or private research centers.
L'archive ouverte pluridisciplinaire HAL, est destinée au dépôt et à la diffusion de documents scientifiques de niveau recherche, publiés ou non, émanant des établissements d'enseignement et de recherche français ou étrangers, des laboratoires publics ou privés. 


\section{DETC2012/VIB-71014}

\section{DESIGN OPTIMISATION OF A NONLINEAR ENERGY SINK EMBEDDED ON A HARMONICALLY FORCED LINEAR OSCILLATOR: THEORETICAL AND EXPERIMENTAL DEVELOPMENTS}

\author{
Etienne Gourc* \\ Université de Toulouse, \\ ICA (Institut Clément Ader), INSA \\ F-31077, Toulouse, FRANCE \\ Sébastien Seguy \\ Université de Toulouse, \\ ICA (Institut Clément Ader), INSA \\ F-31077, Toulouse, FRANCE
}

\author{
Guilhem Michon \\ Université de Toulouse, \\ ICA (Institut Clément Ader), ISAE \\ F-31055, Toulouse, FRANCE \\ Alain Berlioz \\ Université de Toulouse, \\ ICA (Institut Clément Ader), UPS \\ F-31062, Toulouse, FRANCE
}

\begin{abstract}
In this paper, the dynamic response of a harmonically forced Linear Oscillator $(\mathrm{LO})$ strongly coupled to a Nonlinear Energy Sink (NES) is investigated theoretically and experimentally. The system studied comprises a linear oscillator subject to an imposed displacement with an embedded, purely cubic, NES. The behavior of the system is analyzed in the vicinity of $1: 1$ resonance. The complexification averaging technique is used to obtain modulation equations and the associated fixed points. These modulation equations are analyzed using asymptotic expansion to study the regimes related as relaxation oscillation of the slow flow called Strongly Modulated Response (SMR). The zones where SMR occur are computed using a mapping procedure. The Slow Invariant Manifolds (SIM) is used to derive a proper optimization procedure. It is shown that there exist an optimal zone in the parameter plane forcing amplitude-nonlinear stiffness, where SMR occurs without having a high amplitude detached resonance tongue. An experimental setup exhibits a
\end{abstract}

strong mass asymmetry (mass ratio $\approx 1 \%$ ). The cubic stiffness is realized geometrically with two linear spring that extend axially and are free to rotate. Using the previous optimized stiffness of the NES, different frequency response curves and associated zones of SMR are obtained for various forcing amplitude. Good agreement between theoretical and experimental results is observed. The reported experimental results confirm the design procedure, and the possible application of NES for vibration mitigation under periodic forcing.

\section{INTRODUCTION}

In the past decade, it has been demonstrated that addition of small mass, strongly nonlinear attachment to a linear system may give rise, under transient loading, to localization and irreversible transfer of energy, also called pumping. It has been demonstrated that pumping phenomenon can be explained by studying the nonlinear normal modes of the undamped system $[1,2]$. More recent study have introduced suitable asymptotic procedure based on 
the invariant manifold approach to include damping force [3]. Addition of a Nonlinear Energy Sink (NES) changes drastically the dynamic response of the whole system, and may be benefit for vibration mitigation. Energy pumping under transient loading has been widely studied theoretically [4-7] and experimentally [7-9]. In addition to transient loading, system with NES under periodic forcing have also been studied. Steady state response (response with almost constant amplitude) were studied in $[10,11]$ for a grounded NES. Complexification averaging (CXA) technique [12] to derive equation of modulation and compute the fixed points was used. Theses regimes have also been studied analytically and experimentally for an embedded NES in [13]. It has been demonstrated that in addition to weak quasiperiodic response, which are related to Hopf bifurcation of the slow flow, system with NES can exhibit more complex mechanism for vibration mitigation. These regimes are related to relaxation oscillations and are not related to fixed point of the system. When the system exhibit this later type of response (often called Strongly Modulated Response (SMR)), the amplitude of modulation is comparable to the amplitude of the response itself. SMR regimes has been studied in details in $[14,15]$. A design methodology of NES has been proposed in [16] and the result where compared to numerical simulations. The goal of the present paper is to bring experimental developments of energy pumping under periodic forcing and also a design procedure of the NES. The first section is devoted to the theoretical treatment of the equation of motion. An analytic approach is used and the results are compared to numerical simulations. Using these results, a design procedure is presented in section 2 . In section 3 , the experimental results are presented and compared to the theoretical results.

\section{THEORETICAL DEVELOPMENTS}

The theoretical development presented herein are based on $[14,16]$. The system studied in this paper comprises a base excited Linear Oscillator (LO) strongly coupled to a NES (see Fig. 1) and is described by the following set of equation of motion:

$$
\begin{array}{r}
m_{1} \frac{d^{2} x_{1}}{d t^{2}}+c_{1} \frac{d x_{1}}{d t}+c_{2}\left(\frac{d x_{1}}{d t}-\frac{d x_{2}}{d t}\right)+k_{1} x_{1}+ \\
k_{2}\left(x_{1}-x_{2}\right)^{3}=k_{1} x_{e}+c_{1} \frac{d x_{e}}{d t} \\
m_{2} \frac{d^{2} x_{2}}{d t^{2}}+c_{2}\left(\frac{d x_{2}}{d t}-\frac{d x_{1}}{d t}\right)+k_{2}\left(x_{2}-x_{1}\right)^{3}=0
\end{array}
$$

Where $x_{1}, x_{2}, m_{1}, c_{1}, k_{1}$ and $m_{2}, c_{2}, k_{2}$ are the displacement, mass, damping and stiffness of the LO and the NES respectively.

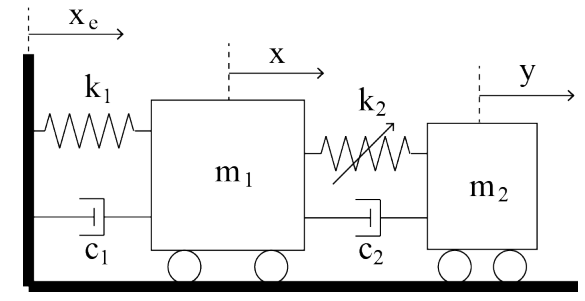

FIGURE 1: SCHEMA OF THE SYSTEM

The imposed harmonic displacement $x_{e}$ is expressed as follow:

$$
x_{e}=G \cos \tilde{\Omega} t
$$

\section{Fixed points}

After rescalling, system $(1,2)$ is reduced to a more convenient form:

$$
\begin{array}{r}
\ddot{x}_{1}+\varepsilon \lambda_{1} \dot{x}_{1}+\varepsilon \lambda_{2}\left(\dot{x}_{1}-\dot{x}_{2}\right)+x_{1}+\varepsilon K\left(x_{1}-x_{2}\right)^{3}= \\
\varepsilon F \cos \Omega \tau-\varepsilon^{2} \lambda_{1} F \omega \sin \Omega \tau
\end{array}
$$

$$
\varepsilon \ddot{x}_{2}+\varepsilon \lambda_{2}\left(\dot{x}_{2}-\dot{x}_{1}\right)+\varepsilon K\left(x_{2}-x_{1}\right)^{3}=0
$$

Where the dots denotes differentiation with respect to $\tau$ and the following paramters are defined: $\tau=\omega_{1} t, \omega_{1}=\sqrt{k_{1} / m_{1}}, \varepsilon=$ $m_{2} / m_{1}, \lambda_{1}=c_{1} / m_{2} \omega_{1}, \Omega=\tilde{\Omega} / \omega_{1}, \lambda_{2}=c_{2} / m_{2} \omega_{1}, \omega_{2}=$ $\sqrt{k_{2} / m_{2}}, K=\omega_{2}^{2} / \omega_{1}^{2}, F=G / \varepsilon$.

New variables are introduced as follow:

$$
v=x_{1}+\varepsilon x_{2}, \quad w=x_{1}-x_{2}
$$

As the system is studied in the vicinity of the $1: 1$ resonance where both oscillators oscillates at the excitation frequency $\Omega$, it convenient to introduce the following complex variables [12]:

$$
\phi_{1} e^{i \Omega \tau}=\dot{v}+i \Omega v, \quad \phi_{2} e^{i \Omega \tau}=\dot{w}+i \Omega w
$$

Introducing Eq $(6,7)$ into $(4,5)$, and keeping only terms containing $e^{i \Omega \tau}$ yields to the following slow modulated system:

$$
\begin{array}{r}
\dot{\varphi}_{1}+\frac{i \Omega}{2} \varphi_{1}+\frac{\varepsilon \lambda_{1}}{2(1+\varepsilon)}\left(\varphi_{1}+\varepsilon \varphi_{2}\right)-\frac{i\left(\varphi_{1}+\varepsilon \varphi_{2}\right)}{2 \Omega(1+\varepsilon)} \\
-\frac{\varepsilon F}{2}-\frac{i \varepsilon^{2} \lambda_{1} F \Omega}{2}=0
\end{array}
$$




$$
\begin{array}{r}
\dot{\varphi}_{2}+\frac{i \Omega}{2} \varphi_{2}+\frac{\varepsilon \lambda_{1}}{2(1+\varepsilon)}\left(\varphi_{1}+\varepsilon \varphi_{2}\right)-\frac{i\left(\varphi_{1}+\varepsilon \varphi_{2}\right)}{2 \Omega(1+\varepsilon)} \\
+\frac{\lambda_{2}(1+\varepsilon)}{2} \varphi_{2}-\frac{3 i K(1+\varepsilon)}{8 \Omega^{3}} \varphi_{2}^{2}\left|\varphi_{2}\right|-\frac{\varepsilon F}{2}-\frac{i \varepsilon^{2} \lambda_{1} F \Omega}{2}=0
\end{array}
$$

A detuning parameter $\sigma$ representing the nearness of the excitation frequency to the natural frequency of the LO is introduced as follow:

$$
\Omega=1+\varepsilon \sigma
$$

Fixed points of Eq $(8,9)$ correspond to periodic solutions of system $(4,5)$. They are computed by equating the derivatives to zero, yielding a system of complex algebraic equations. After algebraic operations, this system is expressed in a more convenient form:

$$
\begin{gathered}
\dot{\varphi}_{1}=\dot{\varphi}_{2}=0 \Rightarrow \varphi_{1}(\tau)=\varphi_{10}, \varphi_{2}(\tau)=\varphi_{20} \\
\varphi_{10}=\frac{\frac{i \varepsilon \varphi_{20}}{(1+\varepsilon)(1+\varepsilon \sigma)}-\frac{\varepsilon^{2} \lambda_{1} \varphi_{20}}{1+\varepsilon}+\varepsilon F+i \varepsilon^{2} \lambda_{1} F(1+\varepsilon \sigma)}{i(1+\varepsilon \sigma)+\frac{\varepsilon \lambda_{1}}{1+\varepsilon}-\frac{i}{(1+\varepsilon)(1+\varepsilon \sigma)}} \\
\alpha_{3} Z_{20}^{3}+\alpha_{2} Z_{20}^{2}+\alpha_{1} Z_{20}+\alpha_{0}=0, \quad Z_{20}=\left|\varphi_{20}\right|^{2}
\end{gathered}
$$

Coefficients $\alpha_{i}(i=1 . .3)$ are not given here due to their length. To study the stability of these fixed points, small perturbations are introduced:

$$
\varphi_{1}=\varphi_{10}+\rho_{1}, \quad \varphi_{2}=\varphi_{20}+\rho_{2}
$$

Keeping only linear terms with respect to $\rho_{i}(i=1 . .2)$, taking the complex conjugate, and putting the resulting system into matrix form, the stability of the fixed points is then deduced by looking at the root of the characteristic equation. If one of the root have a positive real part, the fixed point is unstable, and is stable otherwise.

\section{Asymptotic analysis}

As the case of small mass ratio $(\varepsilon<<1)$ is studied here, $\mathrm{Eq}(8,9)$ is analyzed using a perturbation method. Multiple scales expansion is introduced as follows:

$$
\begin{aligned}
\varphi_{i} & =\varphi_{i}\left(\tau_{0}, \tau_{1}, \ldots\right), \quad \frac{d}{d \tau}=\frac{\partial}{\partial \tau_{0}}+\varepsilon \frac{\partial}{\partial \tau_{1}}+\ldots \\
\tau^{k} & =\varepsilon^{k} \tau, \quad k=0,1, \ldots
\end{aligned}
$$

Substituting Eq (10) and (14) into Eq $(8,9)$ and equating coefficients of like power of $\varepsilon$ yields:

$$
\begin{aligned}
\varepsilon^{0}: \frac{\partial}{\partial \tau_{0}} \varphi_{1} & =0 \\
\frac{\partial}{\partial \tau_{0}} \varphi_{2} & +\frac{\lambda_{2}}{2} \varphi_{2}+\frac{i}{2}\left(\varphi_{2}-\varphi_{1}\right)-\frac{3 i K}{8} \varphi_{2}^{2}\left|\varphi_{2}\right|=0
\end{aligned}
$$

$$
\begin{aligned}
\varepsilon^{1}: & \frac{\partial}{\partial \tau_{1}} \varphi_{1}+\frac{i}{2}\left(\varphi_{1}-\varphi_{2}\right)+i \sigma \varphi_{1}+\frac{\lambda_{1}}{2} \varphi_{1}-\frac{F}{2}=0 \\
& \frac{\partial}{\partial \tau_{1}} \varphi_{2}+\frac{i}{2}\left(\varphi_{1}-\varphi_{2}\right)+\frac{i \sigma}{2}\left(\varphi_{1}+\varphi_{2}\right)+\frac{\lambda_{1}}{2} \varphi_{1}+\frac{\lambda_{2}}{2} \varphi_{2} \\
& -\frac{3 i K(1-3 \sigma)}{8} \varphi_{2}^{2}\left|\varphi_{2}\right|-\frac{F}{2}=0
\end{aligned}
$$

The first equation of (15) gives:

$$
\frac{\partial \varphi_{1}}{\partial \tau_{0}}=0 \Rightarrow \varphi_{1}=\varphi_{1}\left(\tau_{1}, \ldots\right)
$$

Substituting Eq (17) into the second equation of (15), fixed points $\Phi\left(\tau_{1}\right)$ depends only on time scale $\tau_{1}$ and obey algebraic equation:

$$
\frac{\lambda_{2}}{2} \Phi+\frac{i}{2} \Phi-\frac{i}{2} \varphi_{1}-\frac{3 i K}{8} \Phi^{2}|\Phi|=0
$$

Equation (18) is solved by taking $\Phi\left(\tau_{1}\right)=N_{2} \mathrm{e}^{i \delta_{2}}$ :

$$
\begin{gathered}
\left|\phi_{1}\right|^{2}=\lambda_{2}^{2} Z_{2}+Z_{2}-\frac{3 K}{2} Z_{2}^{2}+\frac{9 K^{2}}{16} Z_{2}^{3} \\
Z_{2}\left(\tau_{1}\right)=N_{2}\left(\tau_{1}\right)^{2}
\end{gathered}
$$




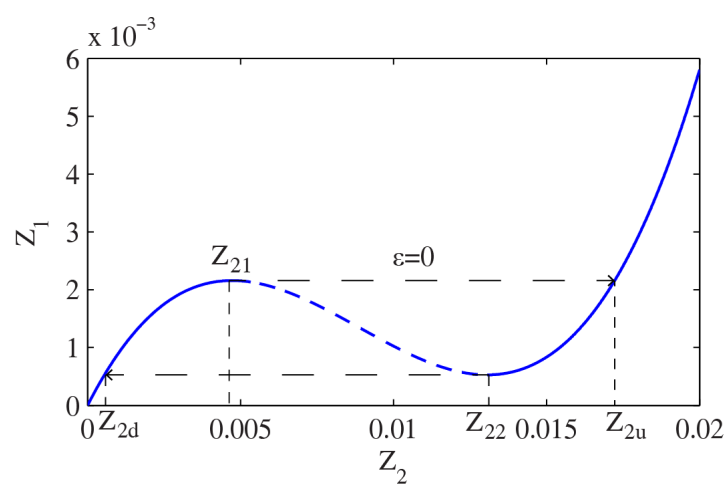

FIGURE 2: EXEMPLE OF SIM FOR $K=100, \lambda_{2}=0.2$.

Number of solutions of Eq. (19) depends only on the parameter $\lambda_{2}$. The roots of the derivative of the right hand side of Eq. (19) are computed to find the critical value of $\lambda_{2}$ :

$$
Z_{2, i}=\frac{4\left(2 \pm \sqrt{1-3 \lambda_{2}^{2}}\right)}{9 K} \quad i=1,2
$$

Therefore, if $\lambda_{2}<1 / \sqrt{3}$, two roots and a pair of saddlenode bifurcation exists and does not exist otherwise. In fact, equation (19) represent the Slow Invariant Manifold (SIM) of the problem. In the case $\lambda_{2}>1 / \sqrt{3}$, the SIM is monotonous. On the opposite, if $\lambda_{2}<1 / \sqrt{3}$, the SIM admits extrema and thus permits to divide the SIM in two stable and one unstable branches. An illustration of the SIM is given in Fig. 2 for $K=100$ and $\lambda_{2}=0.2$. It is well know that such a structure of SIM may give rise to relaxation oscillations. To investigate this possibility, the behavior of Eq. (16) on the SIM is analyzed.

Introducing Eq. (18) into the first equation of (16) yields:

$$
\begin{array}{r}
\frac{\partial}{\partial \tau_{1}}\left[2 i\left(-\frac{\lambda_{2}}{2} \Phi-\frac{i}{2} \Phi+\frac{3 i K}{8} \Phi^{2}|\Phi|\right)\right] \\
+2 i\left(\frac{i}{2}+\sigma i+\frac{\lambda_{1}}{2}\right)\left(-\frac{\lambda_{2}}{2} \Phi-\frac{i}{2} \Phi+\frac{3 i K}{8} \Phi^{2}|\Phi|\right) \\
-\frac{i}{2} \Phi-\frac{F}{2}=0
\end{array}
$$

Expressing $\Phi\left(\tau_{1}\right)$ in polar coordinate, the equations governing the evolution of $N_{2}$ and $\delta_{2}$ with respect to time scale $\tau_{1}$ are obtained:

$$
\frac{\partial N_{2}}{\partial \tau_{1}}=\frac{f_{1}\left(N_{2}, \delta_{2}\right)}{g\left(N_{2}\right)} \quad \frac{\partial \delta_{2}}{\partial \tau_{1}}=\frac{f_{2}\left(N_{2}, \delta_{2}\right)}{g\left(N_{2}\right)}
$$

where

$$
\begin{gathered}
f_{1}\left(N_{2}, \delta_{2}\right)=-9 \lambda_{1} K^{2} N_{2}^{5}+24 \lambda_{1} K N_{2}^{3}-12 F K N_{2}^{2} \cos \delta_{2} \\
-16\left(\lambda_{2}+\lambda_{1}+\lambda_{2}^{2} \lambda_{1}\right) N_{2}+16 F \cos \delta_{2}+16 \lambda_{2} F \sin \delta_{2}
\end{gathered}
$$

$$
\begin{array}{r}
f_{2}\left(N_{2}, \delta_{2}\right)=\left(-54 K^{2} \sigma-27 K^{2}\right) N_{2}^{4}+\left(96 K \sigma+12 K-24 \lambda_{2} \lambda_{1} K\right) N_{2}^{2} \\
+36 K F N_{2} \sin \delta_{2}-16 \lambda_{2}^{2}-32 \sigma-32 \sigma \lambda_{2}^{2} \\
+\frac{16 \lambda_{2} F \cos \delta_{2}-16 F \sin \delta_{2}}{N_{2}}
\end{array}
$$

$$
g\left(N_{2}\right)=54 K^{2} N_{2}^{4}-96 K N_{2}^{2}+32+32 \lambda_{2}^{2}
$$

It has been demonstrated [15] that Eq. (22) admits two types of fixed point. The first type are referred as ordinary fixed points and are found for $f_{1}=f_{2}=0$ and $g \neq 0$. Whereas the others are referred as folded singularities and are found for $f_{1}=f_{2}=g=0$. The system $f_{1}=f_{2}=0$ is written into matrix form:

$$
\left[\begin{array}{ll}
a_{11} & a_{12} \\
a_{21} & a_{22}
\end{array}\right]\left(\begin{array}{c}
\sin \delta_{2} \\
\cos \delta_{2}
\end{array}\right)=\left(\begin{array}{l}
b_{1} \\
b_{2}
\end{array}\right)
$$

with

$$
\begin{aligned}
& a_{11}=16 \lambda_{2} F \text {, } \\
& a_{21}=\frac{36 F K N_{2}^{2}-16 F}{N 2}, \quad a_{22}=\frac{16 \lambda_{2} F}{N_{2}}, \\
& \begin{aligned}
b_{1}= & 9 \lambda_{1} K^{2} N_{2}^{5}-24 \lambda_{1} K N_{2}^{3}+16 N_{2}\left(\lambda_{1}+\lambda_{2}+\lambda_{2}^{2} \lambda_{1}\right), \\
b_{2}= & {\left[27 K^{2} N_{2}^{5}(1+2 \sigma)-12 K N_{2}^{3}\left(1-2 \lambda_{1} \lambda_{2}+8 \sigma\right)\right.} \\
& \left.+16 N_{2}\left(\lambda_{2}^{2}+2 \sigma \lambda_{2}^{2}+2 \sigma\right)\right] / N_{2}
\end{aligned}
\end{aligned}
$$

Ordinary fixed points are found by solving Eq. (26) for $\sin \delta_{2}$ and $\cos \delta_{2}$, and assuming that the determinant does not vanish. It can be noticed that $\operatorname{det}(a)=8 F^{2} g / N_{2}$, so that, eliminating $f_{1}$ and $g$, the condition $f_{2}=0$ is automatically satisfied by Eq. (26), thus obtaining the expression of the folded singularities: 


$$
\begin{array}{r}
\Delta_{i, j}=\gamma_{i} \pm \arccos \left[N _ { 2 , i } \left(16 \lambda_{1}-24 \lambda_{1} K N_{2, i}^{2}+9 \lambda_{1} K^{2} N_{2, i}^{4}+16 \lambda_{2}\right.\right. \\
\left.\left.+16 \lambda_{1} \lambda_{2}^{2}\right) /\left(4 F \sqrt{9 K^{2} N_{2, i}^{4}-24 K N_{2, i}^{2}+16+16 \lambda_{2}^{2}}\right)\right], \\
\gamma_{i}=\arcsin \left(\frac{4 \lambda_{2}}{\sqrt{9 K^{2} N_{2, i}^{4}-24 K N_{2, i}^{2}+16+16 \lambda_{2}^{2}}}\right)
\end{array}
$$

A condition on the excitation amplitude for the existence of folded singularities on the lower and upper fold is obtained from Eq. (28):

$$
\left|\frac{N_{2, i}\left(16 \lambda_{1}-24 \lambda_{1} K N_{2, i}^{2}+9 \lambda_{1} K^{2} N_{2, i}^{4}+16 \lambda_{2}+16 \lambda_{1} \lambda_{2}^{2}\right)}{4 F \sqrt{9 K^{2} N_{2, i}^{4}-24 K N_{2, i}^{2}+16+16 \lambda_{2}^{2}}}\right| \leq 1
$$

Yielding:

$$
F \geq F_{i c}=\frac{N_{2, i}\left(16 \lambda_{1}-24 \lambda_{1} K N_{2, i}^{2}+9 \lambda_{1} K^{2} N_{2, i}^{4}+16 \lambda_{2}+16 \lambda_{1} \lambda_{2}^{2}\right)}{4 \sqrt{9 K^{2} N_{2, i}^{4}-24 K N_{2, i}^{2}+16+16 \lambda_{2}^{2}}}
$$

However, the condition on Eq. (30) is necessary but not sufficient to guaranty the stability of SMR regimes. In effect, under certain condition, the slow flow may be attracted to another stable response. To access this possibility, a procedure of 1D mapping has been developed in [15]. The principle consists in following the slow flow during one cycle of relaxation, with initial condition in the interval $\left[\Delta_{11}, \Delta_{12}\right]$.

The procedure is illustrated in Fig. 3 and consist in four step described bellow:

1. A starting point is chosen on the lower fold (see Eq. (20)) with a phase inside the interval $\left[\Delta_{11}, \Delta_{12}\right]$. The landing points on the upper fold are then computed using Eq. (18) and the invariant property of the SIM (see Fig. 2 for the corresponding notation):

$$
\begin{aligned}
& -\frac{1}{2} \lambda_{2} \Phi_{2,1}-\frac{1}{2} i \Phi_{2,1}+\frac{3}{8} i K \Phi_{2,1}^{2}\left|\Phi_{2,1}\right| \\
= & -\frac{1}{2} \lambda_{2} \Phi_{2, u}-\frac{1}{2} i \Phi_{2, u}+\frac{3}{8} i K \Phi_{2, u}^{2}\left|\Phi_{2, u}\right|
\end{aligned}
$$

2. Equations (22) is integrated numerically with $\Phi_{2 u}$ as initial conditions, until reaching the upper fold line.

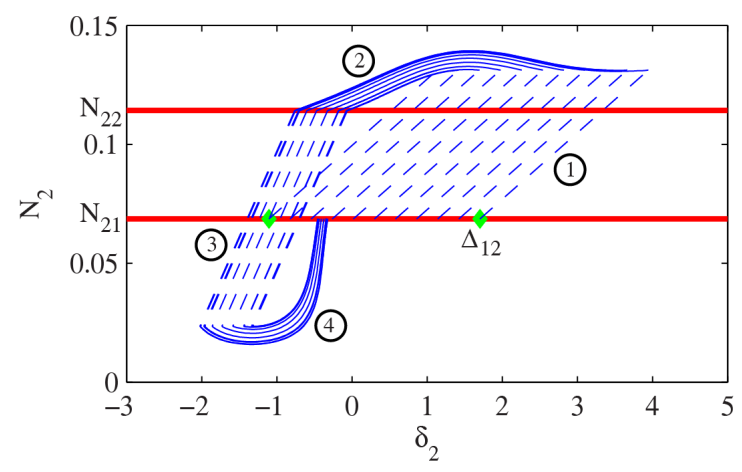

FIGURE 3: ILLUSTRATION OF THE 1D MAPPING PROCEDURE FOR PARAMETERS $K=100, \sigma=1, F=0.15$, $\varepsilon=0.01, \lambda_{1}=0.1, \lambda_{2}=0.2$.

3. The landing point $\Phi_{2 d}$ on the lower fold is computed with the same manner as in the first step.

4. Equations (22) is numerically integrated again until reaching the lower fold line.

This procedure is repeated for various starting points inside the interval $\left[\Delta_{11}, \Delta_{12}\right]$. Finally, if all the points, at the end of step 4 , come back into this interval, the SMR cycle is stable. On the other case, if the slow flow goes through the basin of attraction of a stable fixed point, the SMR cycle is unstable.

\section{NES OPTIMISATION}

All the previous analytic treatments permit to investigate the behavior of the system for vibration mitigation. A complete frequency response function is presented in Fig. 4. Blue dots and stars denote stables and unstable fixed points computed using Eq $(11,12)$. The two bold red vertical lines represent the zone where SMR occurs (i.e. where energy pumping occurs) and are computed using the mapping procedure. The green circles and diamonds represent the root mean square (RMS) value of steady state and SMR response obtained via numerical integration. Note that all frequency response curves in this paper are expressed in RMS value to represent the potential benefits of SMR. Analyti$\mathrm{cal}$ and numerical results are in full agreement. From Fig. 4, it is easy to see that even if the frequency response curve is flattened, there exists a high detached resonance tongue before the natural frequency of the LO, which must absolutely be avoided.

To study the condition of appearance of this detached resonance curve, boundary of zones separating single and triple solutions in Eq. (12), corresponding to saddle node bifurcation, are analyzed. Saddle node bifurcation arises when a real root of the characteristic polynomial quit the left half complex plane. Setting the root equal to 0 , the resulting equation is written in the following form: 

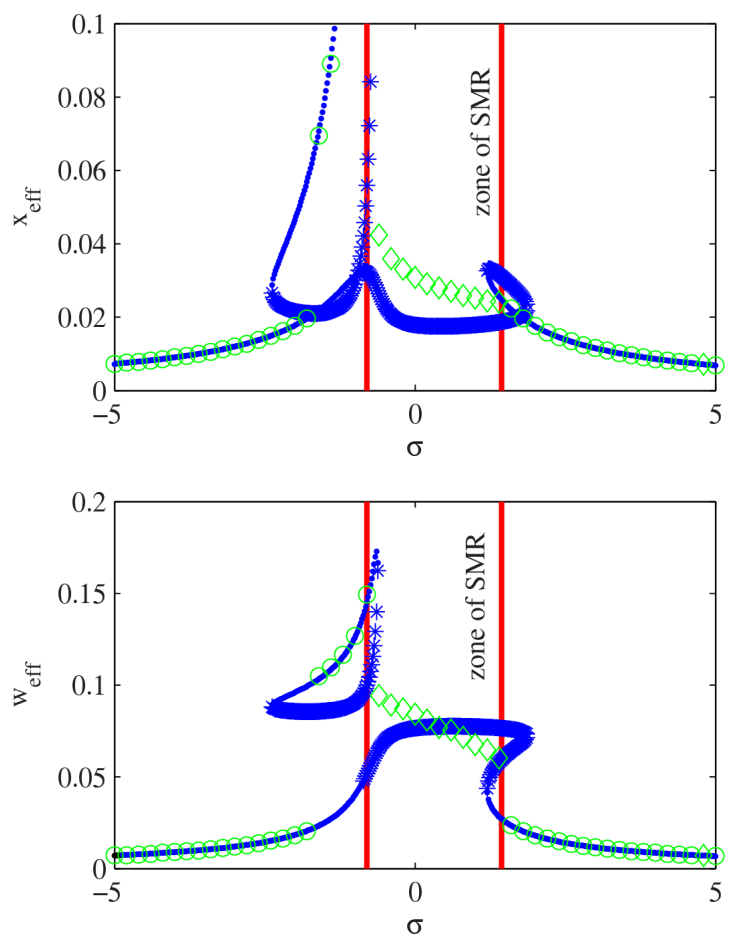

FIGURE 4: ANALYTICAL (Blue) AND NUMERICAL (Green) FREQUENCY RESPONSE FUNCTION FOR PARAMETERS $K=100, \sigma=1, F=0.1, \varepsilon=0.01, \lambda_{1}=0.1, \lambda_{2}=0.2$.

$$
v_{2} Z_{20}^{2}+v_{1} Z_{20}+v_{0}=0
$$

Coefficients $v_{i}$ are not given here due to their length. Eliminating $Z_{20}$ from Eq. (32) yields:

$$
Z_{20}=\frac{-v_{1} \pm \sqrt{v_{1}^{2}-4 v_{2} v_{0}}}{2 v_{2}}
$$

Substituting Eq. (33) into Eq. (12) and solving for $F$ yields to the boundary of zone separating simple to multiple solution in the plane of parameters $(F, \sigma)$. An illustration is given in Fig. 5 .

Fig. 5 highlights that there exist a narrow zone $\left(F_{1 c}<F<\right.$ $\left.F_{S N}\right)$ where energy pumping may be possible $\left(F>F_{1 c}\right)$ and where no high amplitude detached resonance curve exists. This zone is optimal for passive control of vibration using a NES. Taking arbitrary values for $\varepsilon, \lambda_{1}$ and $\lambda_{2}$, the boundary for optimal NES sizing are plotted in the plane of parameters $(F, K)$ in Fig. 6.

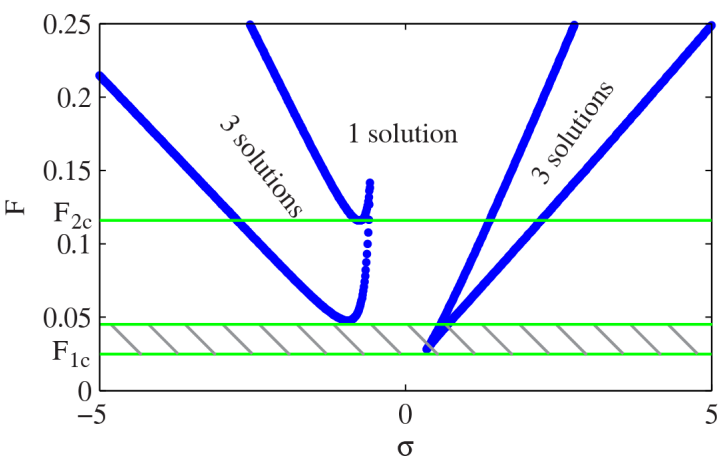

FIGURE 5: BOUNDARY OF THE SADDLE-NODE BIFURCATION FOR PARAMETERS $K=100, \sigma=1, \varepsilon=0.01$, $\lambda_{1}=0.1, \lambda_{2}=0.2$.

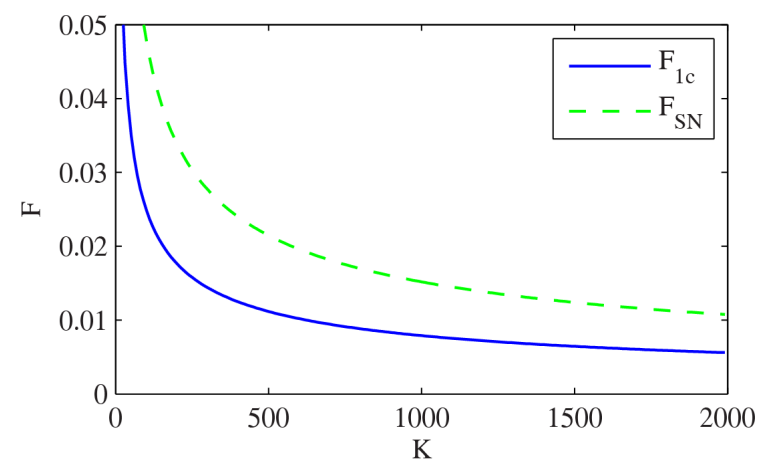

FIGURE 6: CRITICAL FORCING AMPLITUDE AS A FUNCTION OF THE NONLINEAR STIFFNESS $\varepsilon=0.01, \lambda_{1}=0.1$, $\lambda_{2}=0.2$.

In Fig. 7, the evolution of the zone of SMR as a function of the forcing amplitude $F$ is plotted and shows that the closer the working point is from the upper bound $\left(F_{S N}\right)$, the larger will be the zone of SMR. In the next section the experimental setup designed using the previous theoretical developments is presented.

\section{EXPERIMENTAL DEVELOPMENTS}

The experimental setup designed to investigate the behavior of a nonlinear energy sink under harmonic forcing is depicted in Fig. 8. It consists of a single degree of freedom oscillator connected to the frame with a linear spring. On this linear system is embedded a NES connected to the LO with an essential cubic stiffness. Both oscillators are mounted on linear rail guides. The whole set-up is embedded on a $10 \mathrm{kN}$ elecrodynamic shaker. A feedback position control of the electrodynamic shaker ensures a constant excitation amplitude (especially during SMR response 


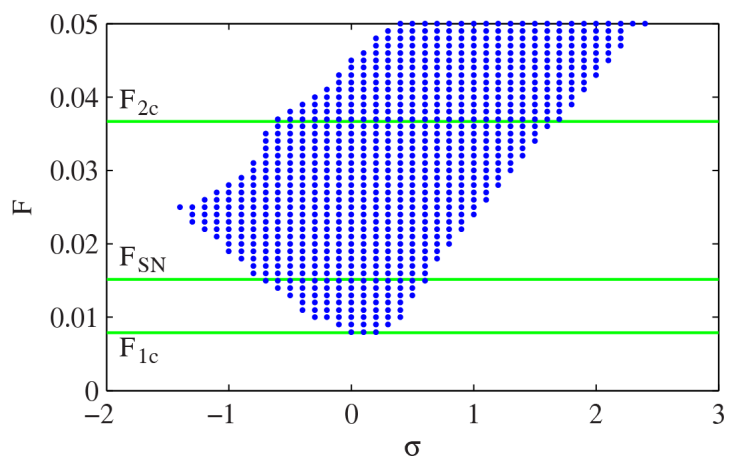

FIGURE 7: ZONE OF SMR AS A FUNCTION OF THE FORCING AMPLITUDE FOR $K=1000, \varepsilon=0.01, \lambda_{1}=0.1, \lambda_{2}=0.2$.

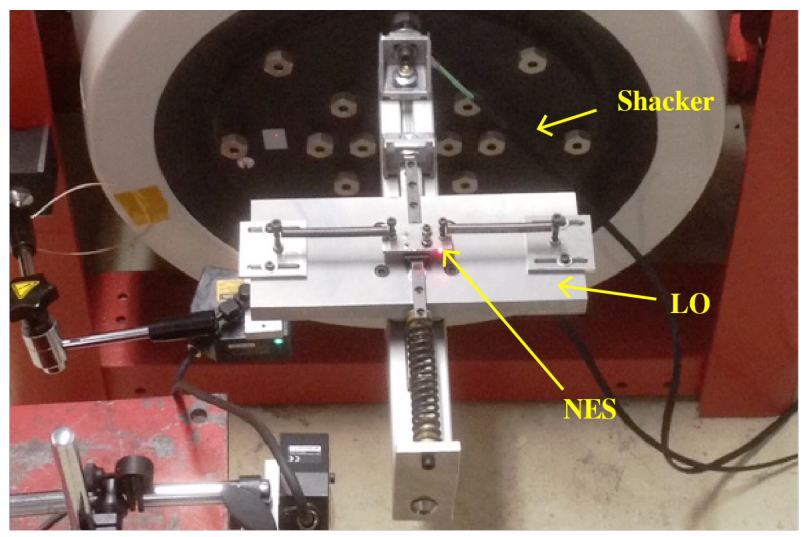

FIGURE 8: GENERAL VIEW OF THE EXPERIMENTAL SETUP

regimes). Displacements of the LO is measured using a laser displacement sensor, and the displacement of the NES using a laser vibrometer.

Raw sensors signals are recorded using a numeric oscilloscope and a band pass filter is applied to correct biases and suppress high frequency noise.

The experimental tests consists of two sets of measurements to obtain the frequency response curves for two different forcing amplitudes. The frequency step is small in the vicinity of the natural frequency of the LO to precisely identify the zone of SMR.

The cubic stiffness restoring force is realized geometrically with two linear springs that extend axially and are free to rotate. The force - displacement relationship is as follows:

$$
f=2 k_{l} w+\frac{2 w\left(P-l \cdot k_{l}\right)}{\sqrt{l^{2}+w^{2}}} \approx \frac{2 P}{l} w+\frac{k_{l}-P}{l^{3}} w^{3}+O\left(u^{5}\right) .
$$

Where $k_{l}$ and $l$ are the stiffness and the length of the springs, and $P$ is the initial pretension. Special care is given to reduce the preload in the springs to reduce the linear stiffness component to a quasi zero value.

The moving mass of the LO and the NES are: $m_{1}=4178 g$ and $m_{N E S}=42 g$. As the mass of the NES is small, the inertia of the springs is no more negligible and has been taken into account. In a rough approximation, considering the spring as a beam, and neglecting axial inertia, the kinetic energy of the NES is written as follows:

$$
T_{N E S}=\int_{0}^{l_{0}} \rho_{S}\left(\frac{x}{l_{0}} \dot{y}\right)^{2} d x+\frac{1}{2} m_{2} \dot{y}^{2}
$$

Where $\rho_{S}=m_{S} / l_{0}$ is the mass density of the spring. Thus the considered moving mass of the NES is $m_{2}=m_{S} / 3+m_{N E S} / 2$. The natural frequency and the viscous damping factor of the main linear system is estimated by performing modal analysis without the NES. The damping coefficient of the linear guide of the NES is also been estimated by removing the nonlinear stiffness, adding a linear spring and performing modal analysis. The friction in the spring's attachment is neglected. All the analyses parameters are summarized below:

$$
\begin{aligned}
m_{1}=4178 g, m_{2} & =42+10=52 g, f_{1}=8.4 \mathrm{~Hz}, \\
c_{1} & =3.97 \mathrm{Ns} / \mathrm{m}, c_{2}=0.36 \mathrm{Ns} / \mathrm{m} .
\end{aligned}
$$

And the associated adimensionalized parameters used for simulation:

$$
\varepsilon=1.2 \%, \lambda_{1}=1.45, \lambda_{2}=0.13 \text {. }
$$

The nominal excitation amplitude is fixed to define the stiffness of the spring composing the NES, that is $G=0.25 \mathrm{~mm}$ $(F=0.02)$. The sizing curves corresponding to the physical parameters of the system are presented in Fig. 9.

The red horizontal line corresponds to the adimensionalized nominal forcing amplitude, and the two black dots correspond to the excitation amplitude presented herein, at the chosen stiffness, that is $K=1874$. The measured force-displacement relationship as well as the cubic fitting are presented in Fig. 10. 


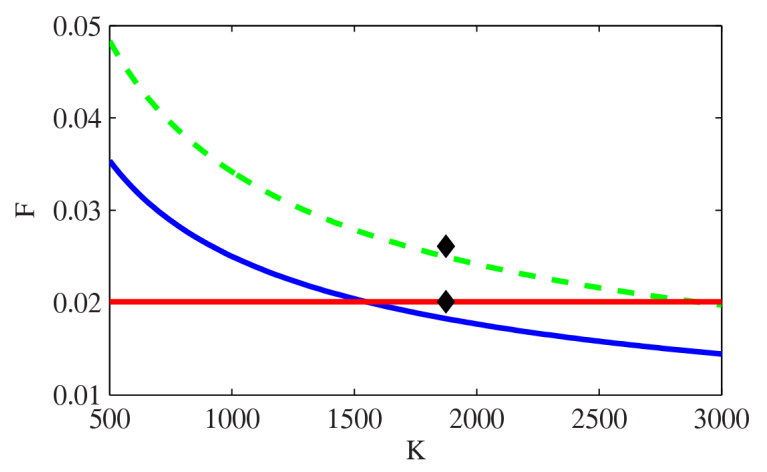

FIGURE 9: DESIGN CURVE CORRESPONDING TO PHYSICAL PARAMETERS.

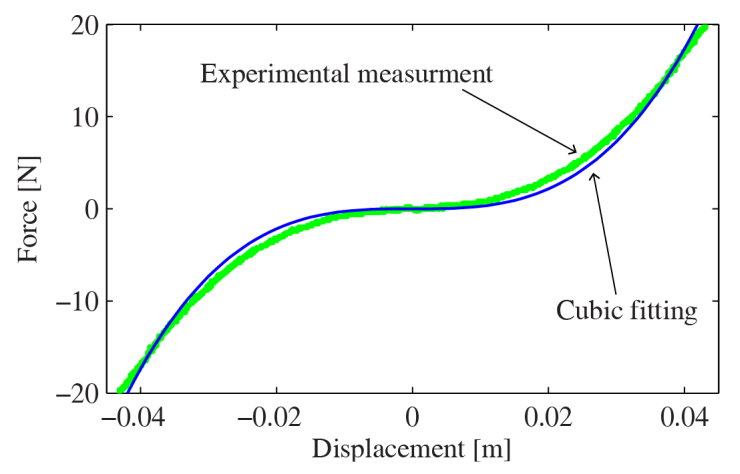

FIGURE 10: FORCE DISPLACEMENT RELATIONSHIP OF THE DESIGNED NES.

The first experiments carried at the nominal forcing amplitude $(G=0.25 \mathrm{~mm})$ is presented in Fig. 11. The analytical frequency response function is presented in blue, and the experimental one in green. The gray dashed and red line represents the theoretical and experimental zone of SMR.

Experimentally, energy pumping through SMR is observed for this forcing amplitude and time response is presented in Fig. 12. It is clear that this regime is related to relaxation oscillation. For this forcing amplitude, no detached resonance curve is observed.

Fig. 13 shows the obtained frequency response curve for $G=0.325 \mathrm{~mm}$. Energy pumping still occurs, but high vibrations amplitudes before the natural frequency are also observed. This is in accordance with the analytical predictions in Fig. 9. The width of SMR zone is also larger when the excitation amplitude increases, as reported theoretically in Fig. 7.

Previous results highlights discrepancies between theoretical prediction and experimental measurements on the zone of
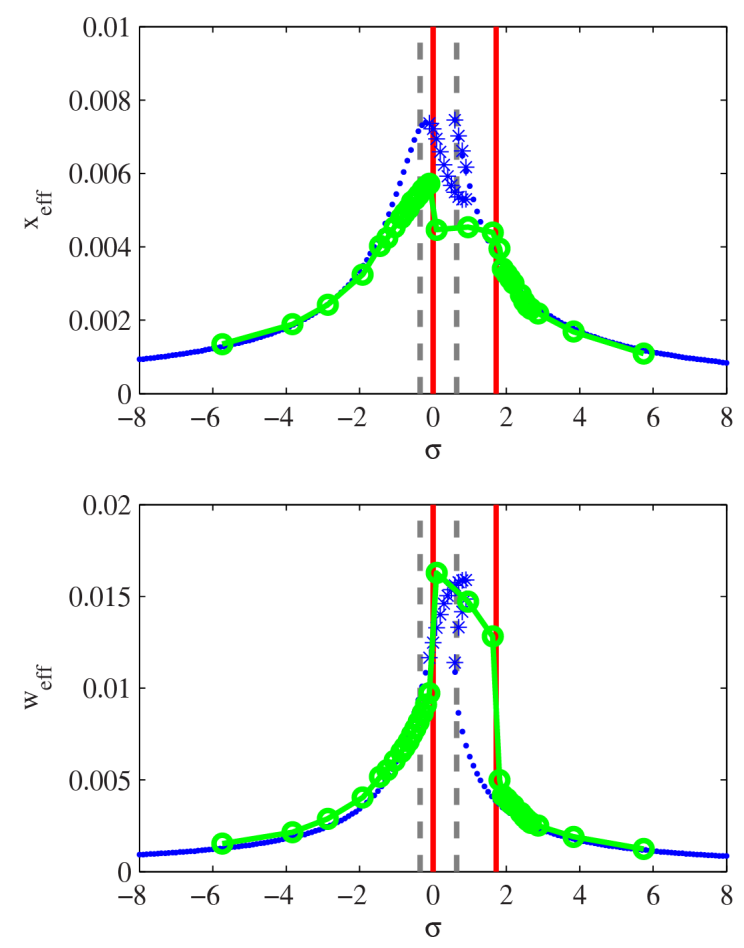

FIGURE 11: EXPERIMENTAL (Green) AND ANALYTICAL (Blue) FRF FOR $G=0.25 \mathrm{~mm}$.
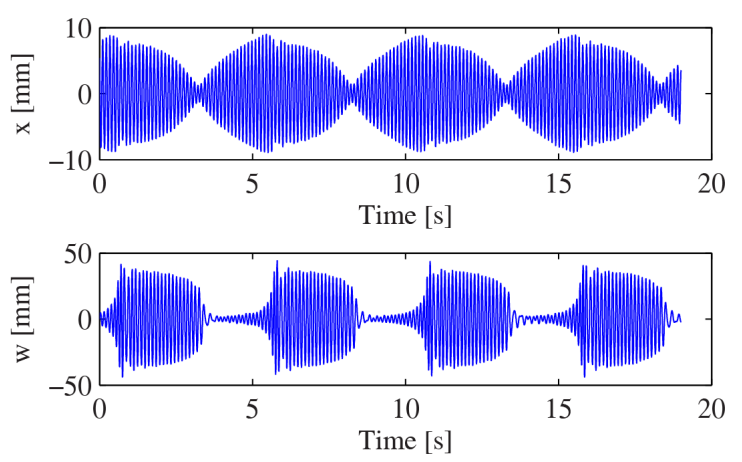

FIGURE 12: EXPERIMENTAL TME RESPONSE OF SMR FOR $G=0.25 \mathrm{~mm}, f=8.5 \mathrm{~Hz}$

SMR. Numerical simulations, have revealed that this zone is sensitive to the damping of both LO and NES, which can explain the difference in the width of the zone. It is observed also that in the both cases, SMR zones are shifted on the left. This is certainly due to a nonlinearity induced in the linear spring anchorage of the LO. However, the behavior observed experimentally shows that energy pumping, under harmonic excitation, is possible without 

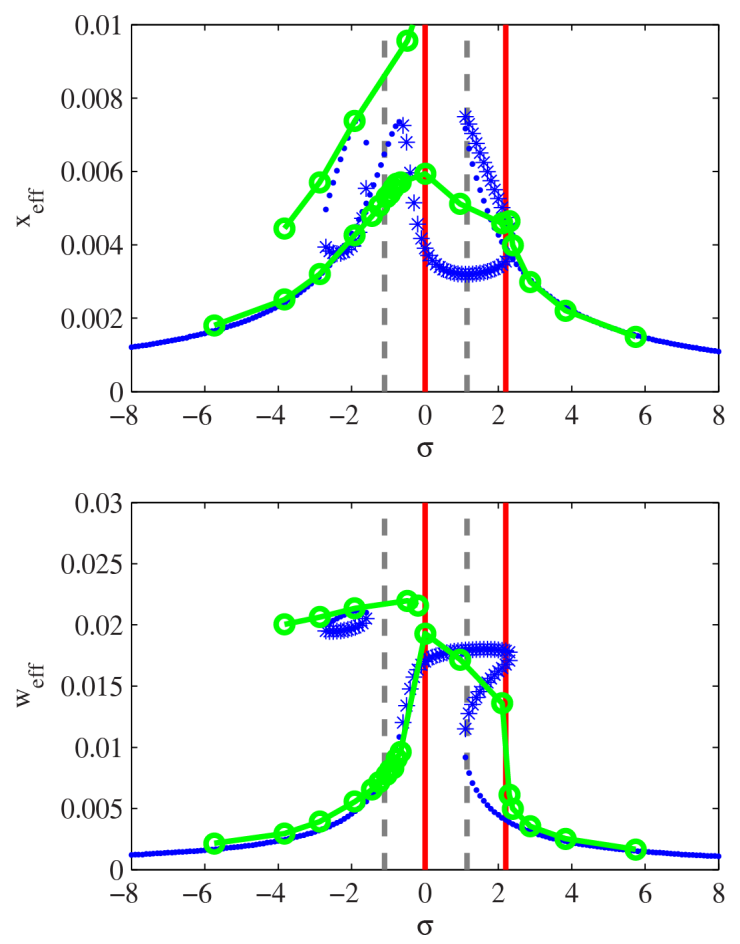

FIGURE 13: EXPERIMENTAL (Green) AND ANALYTICAL (Blue) FRF FOR $G=0.325 \mathrm{~mm}$.

having high amplitude detached resonance tongue. Despite the small mass ratio, the NES induced strong changes in the behavior of the main linear system, and the qualitative behavior of the system is fully explained by the theoretical study.

\section{CONCLUSION}

In this paper, the behavior of an harmonically excited linear oscillator with an embedded NES is investigated theoretically and experimentally. The analysis mainly focused on strongly modulated response which results on strong energy exchange between LO and NES. Theoretical analysis permits to obtain an optimal safe zone for vibration mitigation in the space of parameters $(F, K)$. An experimental set-up has been build based on previous theoretical analysis. The main characteristic of this experimental setup is the low mass ratio $(\varepsilon=1.2 \%)$. Frequency response curves are presented for two different excitation amplitudes. At both excitation amplitude, energy pumping by mean of strongly modulated response has been observed. The experiment also shows that it is possible to avoid the detached resonance curve and always performing energy pumping, validating the design methodology. Even if the comparison between theoretical and experimental results presents some discrepancies, and the design of the experiment is quite simple, repeated trials (not shown here) proved that the system is deterministic.

The use of NES under harmonic excitation could be useful as safety device due to the lack of preferential linear frequency, however the behavior is strongly influenced by damping, and therefore, design with harnessing damping characteristics will be examined in subsequent studies.

\section{REFERENCES}

[1] Gendelman, O., Manevitch, L. I., Vakakis, A. F., and M'Closkey, R., 2001. "Energy pumping in nonlinear mechanical oscillators: Part i-dynamics of the underlying hamiltonian systems". Journal of Applied Mechanics, 68(1), pp. 34-41.

[2] Vakakis, A. F., and Gendelman, O., 2001. "Energy pumping in nonlinear mechanical oscillators: Part ii-resonance capture". Journal of Applied Mechanics, 68(1), pp. 42-48.

[3] Gendelman, O. V., 2004. "Bifurcations of nonlinear normal modes of linear oscillator with strongly nonlinear damped attachment". Nonlinear Dynamics, 37, pp. 115-128.

[4] Quinn, D. D., Gendelman, O., Kerschen, G., Sapsis, T. P., Bergman, L. A., and Vakakis, A. F., 2008. "Efficiency of targeted energy transfers in coupled nonlinear oscillators associated with 1:1 resonance captures: Part i'. Journal of Sound and Vibration, 311(3-5), pp. 1228 - 1248.

[5] Sapsis, T., Vakakis, A., Gendelman, O., Bergman, L., Kerschen, G., and Quinn, D., 2009. "Efficiency of targeted energy transfers in coupled nonlinear oscillators associated with 1:1 resonance captures: Part ii, analytical study". Journal of Sound and Vibration, 325(1-2), pp. 297 - 320.

[6] Kerschen, G., Gendelman, O., Vakakis, A. F., Bergman, L. A., and McFarland, D. M., 2008. "Impulsive periodic and quasi-periodic orbits of coupled oscillators with essential stiffness nonlinearity". Communications in Nonlinear Science and Numerical Simulation, 13(5), pp. 959 - 978.

[7] Gourdon, E., Alexander, N., Taylor, C., Lamarque, C., and Pernot, S., 2007. "Nonlinear energy pumping under transient forcing with strongly nonlinear coupling: Theoretical and experimental results". Journal of Sound and Vibration, 300(3-5), pp. $522-551$.

[8] McFarland, D. M., Bergman, L. A., and Vakakis, A. F., 2005. "Experimental study of non-linear energy pumping occurring at a single fast frequency". International Journal of Non-Linear Mechanics, 40(6), pp. 891 - 899.

[9] Kerschen, G., McFarland, D. M., Kowtko, J. J., Lee, Y. S., Bergman, L. A., and Vakakis, A. F., 2007. "Experimental demonstration of transient resonance capture in a system of two coupled oscillators with essential stiffness nonlinearity". Journal of Sound and Vibration, 299(4-5), pp. 822 838.

[10] Jiang, X., McFarland, D. M., Bergman, L. A., and Vakakis, A. F., 2003. "Steady state passive nonlinear energy pump- 
ing in coupled oscillators: Theoretical and experimental results". Nonlinear Dynamics, 33, pp. 87-102.

[11] Malatkar, P., and Nayfeh, A., 2007. "Steady-state dynamics of a linear structure weakly coupled to an essentially nonlinear oscillator". Nonlinear Dynamics, 47, pp. 167-179.

[12] Manevitch, L. I., 2001. "The description of localized normal modes in a chain of nonlinear coupled oscillators using complex variables". Nonlinear Dynamics, 25, pp. 95-109.

[13] Gourc, E., Michon, G., Seguy, S., and Berlioz, A., 2011. "Experimental investigation and theoretical analysis of a nonlinear energy sink under harmonic forcing". In proceedings of ASME, IDECT.

[14] Starosvetsky, Y., and Gendelman, O., 2008. "Response regimes of linear oscillator coupled to nonlinear energy sink with harmonic forcing and frequency detuning". Journal of Sound and Vibration, 315(3), pp. $746-765$.

[15] Starosvetsky, Y., and Gendelman, O., 2008. "Strongly modulated response in forced 2dof oscillatory system with essential mass and potential asymmetry". Physica D: Nonlinear Phenomena, 237(13), pp. 1719 - 1733.

[16] Starosvetsky, Y., and Gendelman, O., 2008. "Attractors of harmonically forced linear oscillator with attached nonlinear energy sink. ii: Optimization of a nonlinear vibration absorber". Nonlinear Dynamics, 51, pp. 47-57. 